\title{
Antitumor Activity of Thermosensitive Hydrogels Packaging Gambogic Acid Nanoparticles and Tumor-Penetrating Peptide iRGD Against Gastric Cancer
}

This article was published in the following Dove Press journal:

International Journal of Nanomedicine

\author{
Dinghu Zhang ${ }^{1,2, *}$ \\ Yanhong Chu ${ }^{1, *}$ \\ Hanqing Qian (D) \\ Lingyu Qian' \\ Jie Shao' \\ Qiuping $X u^{\prime}$ \\ Lixia Yu' \\ Rutian $\mathrm{Li}^{1}$ \\ Quanan Zhang ${ }^{3}$ \\ Fenglei $\mathrm{Wu}^{4}$ \\ Baorui Liu' \\ Qin Liu'
}

'The Comprehensive Cancer Centre of Drum Tower Hospital, Medical School of Nanjing University and Clinical Cancer Institute of Nanjing University, Nanjing, People's Republic of China; ${ }^{2}$ Department of Oncology, Tongde Hospital of Zhejiang Province, Hangzhou, People's Republic of China; ${ }^{3}$ Department of Oncology, Jiangning Hospital, Nanjing, People's Republic of China; ${ }^{4}$ Department of Oncology, Affiliated Lianyungang Hospital of Xuzhou Medical University, Lianyungang, People's Republic of China

*These authors contributed equally to this work

Correspondence: Qin Liu; Baorui Liu The Comprehensive Cancer Centre of Drum Tower Hospital, Medical School of Nanjing University and Clinical Cancer Institute of Nanjing University, 32I Zhongshan Road, Nanjing 210008,

People's Republic of China

Tel +86-25-8310708I

Fax +86-25-83317016

Email liuqinxh@I26.com;

baoruiliu@nju.edu.cn
Introduction: Gambogic acid (GA) is proved to have anti-tumor effects on gastric cancer. Due to poor solubility, non-specific biological distribution, toxicity to normal tissues and short half-life, it is hard to be applied into the clinic. To overcome these issues, we developed a thermosensitive and injectable hydrogel composed of hydroxypropyl cellulose, silk fibroin and glycerol, with short gelling time, good compatibility and sustained release, and demonstrated that the hydrogel packaged with gambogic acid nanoparticles (GA-NPs) and tumorpenetrating peptide iRGD could improve the anti-tumor activity.

Methods: The Gelling time and micropore size of the hydrogels were regulated through different concentrations of glycerol. Controlled release characteristics of the hydrogels were evaluated with a real-time near-infrared fluorescence imaging system. Location of nanoparticles from different carriers was traced by confocal laser scanning microscopy. The in vivo antitumor activity of the hydrogels packaging GA-NPs and iRGD was evaluated by investigating tumor volume and tumor size.

Results: The thermo-sensitive properties of hydrogels were characterized by $3-4 \mathrm{~min}, 37^{\circ} \mathrm{C}$, when glycerol concentration was $20 \%$. The hydrogels physically packaged with GA-NPs and iRGD showed higher fluorescence intensity than other groups. The in vivo study indicated that the co-administration of GA-NPs and iRGD by hydrogels had higher antitumor activity than the GA-loaded hydrogels and free GA combining with iRGD. Free GA group showed few antitumor effects. Compared with the control group, the body weight in other groups had no obvious change, and the count of leukocytes and hemoglobin was slightly decreased.

Discussion: The hydrogel constructed iRGD and GA-NPs exerted an effective anti-tumor effect possibly due to retention effect, local administration and continuous sustained release of iRGD promoting the penetration of nanoparticles into a deep part of tumors. The delivery system showed little systemic toxicity and would provide a promising strategy to improve anti-gastric cancer efficacy.

Keywords: gambogic acid, hydrogel, nanoparticles, iRGD, gastric cancer

\section{Introduction}

Gastric cancer is the third leading cause of cancer-related mortality worldwide. The low rate of early diagnosis means that most patients have advanced-stage diseases at diagnosis. Therefore, the 5-year survival rate for patients with gastric cancer is below 20\%. ${ }^{1,2}$ Recently, polymer materials have been used in gastric cancer 
treatment. ${ }^{3}$ Polymers, such as hydrogels, possess the ability to reduce severe side effects of chemotherapeutic drugs and many of them are easy to operate and cause minimal trauma. ${ }^{4-7}$

In recent years, silk fibroin hydrogels have been widely utilized in biomedical fields such as tissue regeneration and drug sustained release, owing to their good biocompatibility, thermal stability and controllable mechanical properties. ${ }^{8-10}$ A kind of thermosensitive injectable hydrogel was prepared with hydroxypropyl cellulose (HPC) and silk fibroin (SF). When the mixed solution was heated, the hydrophobicity of HPC gradually increased with the increase of solution temperature, which made SF changing from irregular curling to beta-folding and further made the mixed solution gelled. Although in this way, HPC shortened the gelation time of SF to $40 \mathrm{mins}$, it is still relatively long in practical application.

Gambogic acid (GA) is one of the main components of gamboge, which could have anti-tumor effects in various cancers, such as breast cancer, ${ }^{11}$ gastric cancer, ${ }^{12}$ liver cancer, ${ }^{13}$ and lung cancer. ${ }^{14}$ However, poor solubility, nonspecific biological distribution, toxicity to normal tissues and short half-life limit the further application of GA in the clinic. ${ }^{15,16}$ Recent researches have demonstrated that intravenous injection of nanoparticles loaded with gambogic acid (GA-NPs) has improved the anti-tumor effect of GA. Factors accounting for this are mainly several characteristics of nanoparticles: improved water solubility of drugs, sustained release and enhanced permeability and retention effect (EPR effect). ${ }^{17-19}$ Although intravenous injection can improve the curative effect, it is still hindered by reticuloendothelial system uptake, intertissue pressure, osmotic barrier of tumors, and the accumulation of antineoplastic drugs in tumors.

$\alpha v \beta 3$ integrin receptor and neurociliary protein-1 (NRP1) are overexpressed in tumor tissues and tumor vessels. ${ }^{20-23}$ iRGD (CRGDRCPDC) is a disulfide bond cycle-based RGD peptide. It is capable of binding to $\alpha v \beta 3$ integrin receptor, and through enzymatic hydrolysis of tumour-derived proteins, exposing the binding sequence targeted NRP-1. When iRGD binds to $\alpha v \beta 3$ integrin receptor and NRP-1, the permeability of tumour tissues is increased and more drug molecules or drug-loaded nanoparticles delivered together with iRGD can penetrate into tumour tissues. As a result, the antitumor effect of drugs is apparently improved. ${ }^{24-29}$

In our research, we prepared an injectable thermosensitive hydrogel, consisting of HPC, SF and glycerol (Gly), with good sustained-release properties and made the hydrogels loaded with GA-NPs and iRGD. We assumed that when the hydrogels with GA-NPs and iRGD were injected around the tumors, continuously sustainedreleased iRGD and the GA-NPs would penetrate into the tumors, enhancing the anti-tumor efficacy of GA.

\section{Materials and Methods Materials}

Hydroxypropyl cellulose (HPC, Mn=100 kDa) was purchased from Alfa Aesar (Shanghai, China). Silkworm cocoons were purchased from Jiangsu, China. Glycerol was purchased from Nanjing WanQing Chemical Glassware Instrument CO.LTD (Jiangsu, China) and used as received. Gambogic acid (99.6\%) and Rhodamine B were purchased from Aladdin (Shanghai, China). iRGD was custom-made by GL Biochem (Shanghai) Ltd (China). PEG $_{2000}-\mathrm{PCL}_{6000}$ was purchased from Jinan Daigang Biomaterial Co., Ltd (China). NIR-797 and 4',6-diamidino-2-phenylindole (DAPI) were obtained from Sigma-Aldrich (USA). Other reagents were used without further purification.

\section{Preparation of Silk Fibroin}

The silkworm cocoons were cut into fragments and boiled in $0.5 \%$ sodium carbonate solution. After about $30 \mathrm{mins}$, they were rinsed with water. Then, the same process was repeated. Processed silkworm cocoons were dried at $50^{\circ} \mathrm{C}$ and added to the mixed solution of calcium chloride/ethanol/water (molar ratio $1: 2: 8$ ), $70^{\circ} \mathrm{C}, 4 \mathrm{~h}$. Afterwards, the mixture was dialyzed in a dialysis bag $(14 \mathrm{KDa})$ for $72 \mathrm{~h}$, followed by centrifugation (10,000 r, $15 \mathrm{~min})$. Finally, through a $0.22-\mu \mathrm{m}$ membrane and freeze-drying, silk fibroin was prepared and preserved at $-20^{\circ} \mathrm{C}$.

\section{Preparation of HPC/SF/Gly Hydrogels}

The mixture of glycerol-water concentration $(\mathrm{v} / \mathrm{v})$ was 0 , $10 \%$ and $20 \%$. SF solution, $140 \mathrm{mg} / \mathrm{mL}$, and $70 \mathrm{mg} / \mathrm{mL}$ HPC solution were prepared using each concentration of glycerol-water mixture. Then, $0.5 \mathrm{~mL} \mathrm{SF}$ solution and $0.5 \mathrm{~mL} \mathrm{HPC}$ solution were mixed to prepare $\mathrm{HPC} / \mathrm{SF} /$ Gly solution. According to the concentration of glycerol, the prepared $\mathrm{HPC} / \mathrm{SF} / \mathrm{Gly}$ mixture was respectively named HPC/SF/Gly0, HPC/SF/Gly10 and HPC/SF/Gly20. These three solutions were heated to $37^{\circ} \mathrm{C}$. The gelation time was measured by tube inversion method: after inversing the tube for $1 \mathrm{~min}$, no liquid flowing out means gelation. These three hydrogels were then freeze-dried and characterized by scanning electron microscopy (SEM, JSM5410LV, JEOL, Japan). 


\section{Degradability of HPC/SF/Gly Hydrogels}

Male ICR mice, 10-12 weeks old, were purchased from the Yangzhou University Animal Resource Center. All animal experiments were performed in full compliance with guidelines in the Guide for the Care and Use of Laboratory Animals and were approved by the Ethics Review Board for Animal Studies of Nanjing Drum Tower Hospital, Medical School of Nanjing University. Two hundred microliters $\mathrm{HPC} / \mathrm{SF} / \mathrm{Gly}$ hydrogels (20\% glycerol concentration) were injected subcutaneously into ICR mice. At different time points, mice were sacrificed to observe the degradation of hydrogels and the inflammation around.

\section{In vivo Near-Infrared Imaging}

Male BALB/c nude mice, 6-8 weeks old, were purchased from the Yangzhou University Animal Resource Center. All animal experiments were performed in full compliance with guidelines in the Guide for the Care and Use of Laboratory Animals and were approved by the Ethics Review Board for Animal Studies of Nanjing Drum Tower Hospital, Medical School of Nanjing University. A $0.1 \mathrm{~mL}$ NIR797 solution $(0.02 \mathrm{mg} / \mathrm{mL})$ or $\mathrm{HPC} / \mathrm{SF} /$ Gly solution (concentration of glycerol: 20\%) loaded with equivalent NIR797 were injected into nude mice subcutaneously. The fluorescence signals were examined at different time points. Observation was performed on an optical and X-ray small animal imaging system (In-Vivo Xtreme, Bruker).

\section{Preparation of Nanoparticles Loaded with Gambogic Acid (GA-NPs)}

GA-NPs were prepared by the emulsifying method. GA, $8.3 \mathrm{mg}$, and $40 \mathrm{mg} \mathrm{PEG}_{2000}-\mathrm{PCL}_{6000}$ polymer were dissolved in $0.5 \mathrm{~mL}$ dichloromethane solution; then, $1.5 \mathrm{~mL}$ $3 \%$ PVA (w/v) was added. After ultrasound (time: $1 \mathrm{~min}$, power: $30 \mathrm{w}), 2.5 \mathrm{~mL}$ 0.5\% PVA was added and ultrasound (time: $30 \mathrm{~s}$, power: $20 \mathrm{w}$ ). Then, the mixture was stirring in a ventilated kitchen. About $1 \mathrm{~h}$ later, the solution changed from milky white to light blue. After filtered with $0.45-\mu \mathrm{m}$ filter membranes, the morphology of GANPs was observed by transmission electron microscopy (TEM, JEM-100 S, JEOL, Japan). The drug loading and encapsulation efficiency of DOC were analyzed by HighPerformance Liquid Chromatography (HPLC) system.

The drug loading content and encapsulation efficiency were calculated by the following Eqs. (1) and (2), respectively:

$$
\text { Drug loading content } \%=\frac{\begin{array}{l}
\text { Weight of the drug } \\
\text { in nanoparticles }
\end{array}}{\begin{array}{l}
\text { Weight of the } \\
\text { nanoparticles }
\end{array}} \times 100 \%
$$

$$
\text { Encapsulation efficiency } \%=\frac{\begin{array}{l}
\text { Weight of the drug } \\
\text { in nanoparticles }
\end{array}}{\begin{array}{l}
\text { Weight of the } \\
\text { feeding drug }
\end{array}} \times 100 \%
$$

\section{Preparation of Nanoparticles Loaded with NIR797 (NIR797-NPs)}

NiR797-NPs were prepared by the emulsifying method. NIR797, $80 \mu \mathrm{g}$, and $40 \mathrm{mg} \mathrm{PEG}_{2000}-\mathrm{PCL}_{6000}$ polymer were dissolved in $2 \mathrm{~mL}$ DMSO solution, stirred overnight, and then put into a dialysis bag (3500 Da) for 2 days. The liquid in the dialysis bag was then taken out and freeze-dried.

\section{Preparation of Nanoparticles Loaded with Rhodamine B (Rho-NPs)}

Rho-NPs were prepared by the emulsifying method. One milligram Rhodamine $\mathrm{B}$ and $40 \mathrm{mg} \mathrm{PEG}_{2000}-\mathrm{PCL}_{6000}$ polymer were dissolved in $2 \mathrm{~mL}$ DMSO solution, stirred overnight, and then put into a dialysis bag for 2 days. The liquid in the dialysis bag was then taken out and freeze-dried.

\section{Preparation of Hydrogels Loaded with Different Drugs}

Hydrogels loaded with NIR797-NPs (Gel (NIR797-NPs)) were prepared by dissolving $70 \mathrm{mg} \mathrm{SF}$ and $30 \mathrm{mg}$ HPC in a mixture of $700 \mu \mathrm{L}$ NIR797-NPs solution, $100 \mu \mathrm{L}$ water and $200 \mu \mathrm{L}$ glycerol. Hydrogels loaded with NIR797-NPs and iRGD (Gel (NIR797-NPs+iRGD)) were prepared by dissolving $70 \mathrm{mg}$ SF and $30 \mathrm{mg}$ HPC in a mixture of 700 $\mu \mathrm{L}$ NIR797-NPs solution, $100 \mu \mathrm{L}$ iRGD $(10 \mathrm{mg} / \mathrm{mL})$ and $200 \mu \mathrm{L}$ glycerol. Hydrogels loaded with Rho-NPs (Gel (Rho-NPs)) was prepared by dissolving $70 \mathrm{mg}$ SF and $30 \mathrm{mg}$ HPC in a mixture of $700 \mu \mathrm{L}$ Rho-NPs solution, 100 $\mu \mathrm{L}$ water and $200 \mu \mathrm{L}$ glycerol. Hydrogels loaded with RhoNPs and iRGD (Gel (Rho-NPs+iRGD)) were prepared by dissolving $70 \mathrm{mg}$ SF and $30 \mathrm{mg} \mathrm{HPC}$ in a mixture of $700 \mu \mathrm{L}$ Rho-NPs solution, $100 \mu \mathrm{L}$ iRGD $(10 \mathrm{mg} / \mathrm{mL})$ and $200 \mu \mathrm{L}$ glycerol. Hydrogels loaded with GA-NPs (Gel (GA-NPs)) (GA: $0.4 \mathrm{mg} / \mathrm{mL}$ ) were prepared by dissolving $70 \mathrm{mg} \mathrm{SF}$ and $30 \mathrm{mg}$ HPC in a mixture of $700 \mu \mathrm{L}$ GA-NPs solution 
(GA: $0.57 \mathrm{mg} / \mathrm{mL}$ ), $100 \mu \mathrm{L}$ water and $200 \mu \mathrm{L}$ glycerol. Hydrogels loaded with GA-NPs and iRGD (Gel (GA-NPs +iRGD)) (GA: $0.4 \mathrm{mg} / \mathrm{mL}$, iRGD: $1 \mathrm{mg} / \mathrm{mL}$ ) were prepared by dissolving $70 \mathrm{mg}$ SF and $30 \mathrm{mg}$ HPC in a mixture of 700 $\mu \mathrm{L}$ GA-NPs solution (GA: $0.57 \mathrm{mg} / \mathrm{mL}$ ), $100 \mu \mathrm{L}$ iRGD $(10 \mathrm{mg} / \mathrm{mL})$ and $200 \mu \mathrm{L}$ glycerol.

\section{In vitro NPs Uptake Assay}

Human gastric cancer cell line MKN-45 was purchased from the Cell Bank of the Chinese Academy of Sciences (Shanghai, China). The cells were cultured in McCoy's 5A medium (Gibco) containing fetal bovine serum (FBS, $10 \%)$, penicillin $(100 \mathrm{U} / \mathrm{mL})$, and streptomycin $(100 \mathrm{mg} /$ $\mathrm{mL}$ ) and incubated under a $5 \% \mathrm{CO}_{2}$ atmosphere at $37^{\circ} \mathrm{C}$.

One-milliliter, $1 \times 10^{4}$ MNK-45 cells were planted in a confocal dish. After adherence, $50 \mu \mathrm{L}$ Rho-NPs and equivalent Rho-

NPs+iRGD solution (iRGD: $0.1 \mathrm{mg} / \mathrm{mL}$ ) were added into the dish. After $1 \mathrm{~h}$, the supernatant was removed. Then, the dish was washed for three times, formaldehyde fixed and DAPI stained. Confocal imaging of cells was finally performed.

One-milliliter, $1 \times 10^{6}$ MNK-45 cells each well were planted on a 6-well plate. After adherence, $50 \mu \mathrm{L}$ Rho-NPs and equivalent Rho-NPs+iRGD solution (iRGD: $0.1 \mathrm{mg}$ / $\mathrm{mL}$ ) were added into the 6-well plate, respectively. After 1 $\mathrm{h}$, cells were digested by trypsinase and flow cytometry was used to detect the fluorescence intensity of cells.

\section{Noninvasive Detection of Intratumoral Distribution of Fluorescence-Labeled NPs}

Female BALB/c nude mice, 5 weeks old, were purchased from the Yangzhou University Animal Resource Center. All animal experiments were performed in full compliance with guidelines in the Guide for the Care and Use of Laboratory Animals and were approved by the Ethics Review Board for Animal Studies of Nanjing Drum Tower Hospital, Medical School of Nanjing University. $1 \times 10^{6} \mathrm{MKN}-45$ cells were injected subcutaneously under the first mammary gland of nude mice to develop tumors.

NIR797, NIR797-NPs, NIR797-NPs+iRGD, Gel (NIR797-NPs) and Gel (NIR797-NPs +iRGD) (equated to the amount of $0.2 \mathrm{~mL}$ NIR797) were, respectively, injected around the tumors of nude mice and imaging was performed at different time points. After $120 \mathrm{~h}$, the nude mice were sacrificed and the tumors of each group were dissected for imaging.

\section{The Penetration of NPs in Tumor Tissues} Rho, Rho-NPs, Rho-NPs+iRGD, Gel (Rho-NPs) and Gel (Rho-NPs+iRGD) (equated to the amount of $0.2 \mathrm{~mL}$ Rho) were, respectively, injected around the tumors. After $120 \mathrm{~h}$, the tumors were removed to prepare frozen sections. The nuclei were stained with DAPI and the samples were observed under a Zeiss LSM510 Meta confocal microscope.

\section{In vivo Antitumor Effect Assay}

All animal experiments were performed in full compliance with guidelines in the Guide for the Care and Use of Laboratory Animals and were approved by the Ethics Review Board for Animal Studies of Nanjing Drum Tower Hospital, Medical School of Nanjing University. Female $\mathrm{BALB} / \mathrm{c}$ nude mice, 5 weeks old, were purchased from the Yangzhou University Animal Resource Center. Animals were raised under pathogen-free conditions. MKN-45 cells, $1 \times 10^{6}$, were injected subcutaneously under the first mammary gland of nude mice to develop tumors. When the volume of tumors reached about $150 \mathrm{~mm}^{3}$, nude mice were randomly divided into six groups: control group, free GA, GA-NPs, GA-NPs+iRGD, Gel(GA-NPs) and Gel (GA-NPs + iRGD) $(\mathrm{n}=6)$. The dose of GA was $4 \mathrm{mg} / \mathrm{kg}$ and the control group was given saline of equal volume. Tumor volume and mice weight were measured every 3-6 days.

The formula for calculating the volume of tumors is: Volume of tumor $=0.5 \times$ length $\times$ width $^{2}$. The formula for calculating the relative body weight: Relative body weight $=$ absolute body weight of nude mice/absolute body weight of nude mice on the first day of treatment. On the 16th day, the blood of nude mice in each group was extracted for leukocyte and platelet detection. After that, the nude mice were sacrificed and the tumors were weighed and used to make HE and TUNEL staining sections.

\section{Statistical Analysis}

Statistical analyses of data were made by ANOVA analysis (SPSS16.0) and values were considered to be statistically significant if $\mathrm{P}<0.05$.

\section{Results}

\section{Effects of Different Glycerol} Concentration on the Gelation Time and Pore Diameter of HPC/SF/Gly Hydrogels

Herein, we prepared a thermosensitive and injectable hydrogel composed of HPC, SF and glycerol. The gelation time of the hydrogels was regulated through different concentrations 
of glycerol. When a single HPC solution was heated to $37^{\circ} \mathrm{C}$, there were no obvious changes. When glycerol was added $(20 \% \mathrm{v} / \mathrm{v})$ in the HPC solution, the mixture became turbid, indicating that the HPC solution had a phase transition. When the HPC solution with glycerol was mixed with SF solution and heated for 3-4 min, the mixture solution changed from flowing liquid to translucent solid hydrogel (Figure 1A). The gelation time of HPC/SF/Gly hydrogels was about 20-25 min, 13-16 min and 3-4 min when glycerol concentration was $0,10 \%$ and $20 \%$. Such results indicated that the gelation time of HPC/SF/Gly hydrogels was shortened with the increase of glycerol concentration.

Glycerol concentration also has effects on the pore size of hydrogels. The structure of HPC/SF/Gly0 hydrogels, hydrogel without glycerol, is open, circular and interconnected porous, with an average pore diameter of about 12 microns. HPC/SF/Gly10 hydrogels (glycerol: 10\%) still show a porous circular structure, but the pore size is smaller than $\mathrm{HPC} / \mathrm{SF} / \mathrm{Gly} 0$ hydrogels, averaging 5 microns. When $20 \%$ glycerol is added, the pore size of HPC/SF/Gly20 hydrogels was significantly reduced to about $400 \mathrm{~nm}$ (Figure 1B).

\section{Characterization of HPC/SF/Gly Hydrogels Loaded with Nanoparticles}

Water-soluble GA-NPs with 57\% encapsulation efficiency and $9 \%$ drug loading were prepared by the emulsifying method. The particle size of GA-NPs was about $200 \mathrm{~nm}$ (Figure 1C). Figure 1D was HPC/SF/Gly hydrogel loaded with GA-NPs, which showed yellow liquid state at room temperature, and transformed into solid state when at $37^{\circ} \mathrm{C}$ after about 4-5 min. It showed that GA-NPs had no significant effect on the gelation time of $\mathrm{HPC} / \mathrm{SF} / \mathrm{Gly}$ system. Figure $1 \mathrm{E}$ and $\mathrm{F}$ showed the accumulative diffusion profiles of GA through GA-NPs, and of GA and iRGD through Gel (GA-NPs +iRGD) respectively. GA was released more slowly when capsuled with HPC/SF/Gly hydrogel.

As shown in Figure 2A, no apparent HPC/SF/Gly hydrogel degradation was observed in the first 3 weeks. On the 21 st day, the slight inflammatory reaction occurred in the subcutaneous tissue of the hydrogel area. There was a slight congestion of blood vessels, but no local exudate. On the 42nd day, HPC/SF/Gly hydrogel was observed to be degraded, as the gel became thin and the inflammatory responses disappeared. Two months later, there was no obvious degradation of the hydrogel and visible inflammation compared with the 42 nd day. After 4 months, there were almost no hydrogel residues in mice subcutaneously.

The hydrogels loaded with NIR797 and free NIR797 solution were separately subcutaneously injected into nude mice. As shown in Figure 2B, the fluorescence intensity of Free NIR797 group and Gel+NIR797 group decreased with the prolongation of time. While the fluorescence

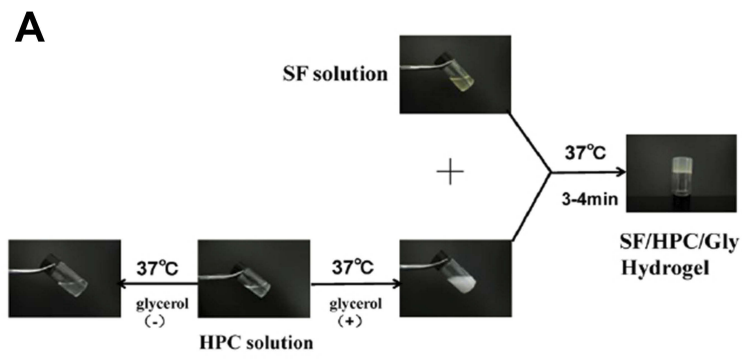

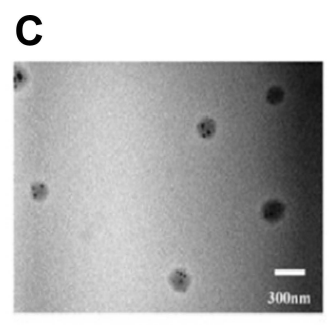

GA-NPs

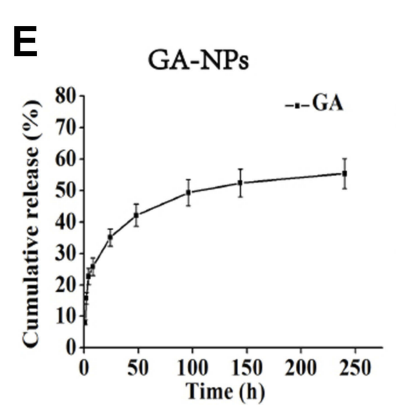

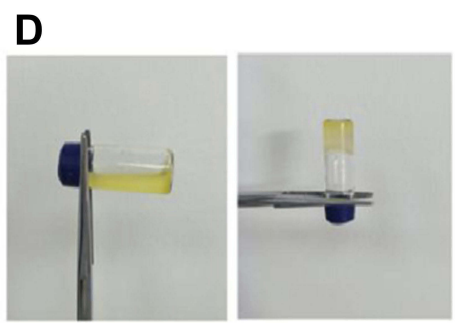

Gel(GA-NPs)

$\mathbf{F}$

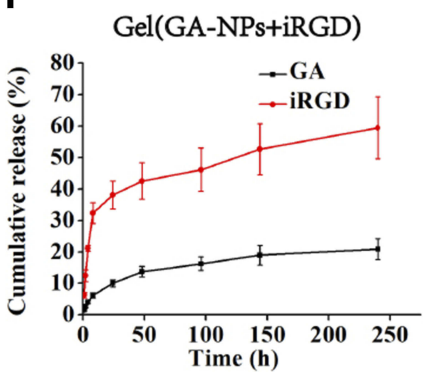

Figure I Characterization of hydrogels and nanoparticles. (A) Gel-forming schematic diagram of HPC/SF/Gly hydrogel. (B) SEM of HPC/SF/Gly hydrogels (Scale Bar=25 $\mu$ m, $15 \mu \mathrm{m}, 5 \mu \mathrm{m}, 600 \mathrm{~nm}$ ). (C) SEM of GA-NPs (Scale Bar=300 nm). (D) Photographs of Gel (GA-NPs) at different temperatures $\left(25^{\circ} \mathrm{C}, 37^{\circ} \mathrm{C}\right)$. (E) Accumulative diffusion profiles of GA through GA-NPs. (F) Accumulative diffusion profiles of GA and iRGD through Gel (GA-NPs +iRGD).

Abbreviations: HPC, hydroxypropyl cellulose; SF, silk fibroin; Gly, glycerol; GA-NPs, nanoparticles loaded with gambogic acid; Gel (GA-NPs), Hydrogels loaded with GANPs. 


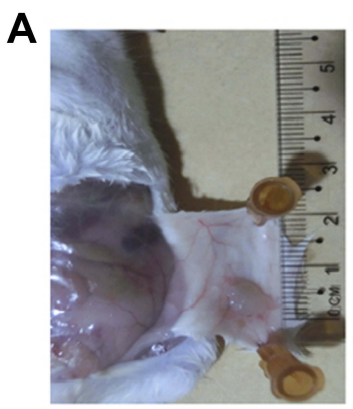

Day 9

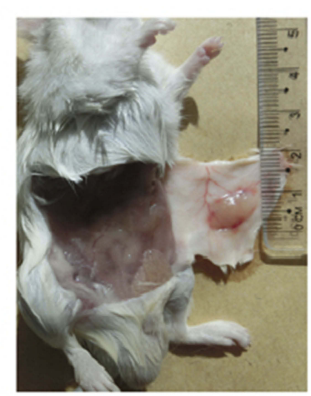

Day 21

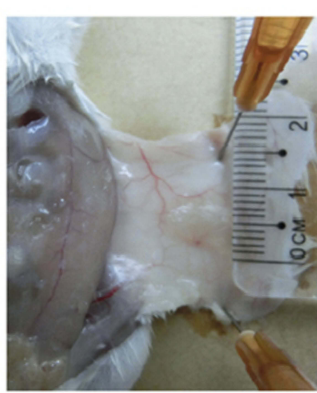

Day 42

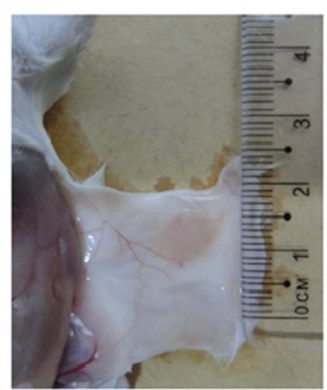

Day 60

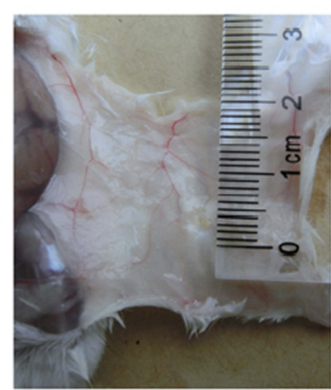

Day 120

B

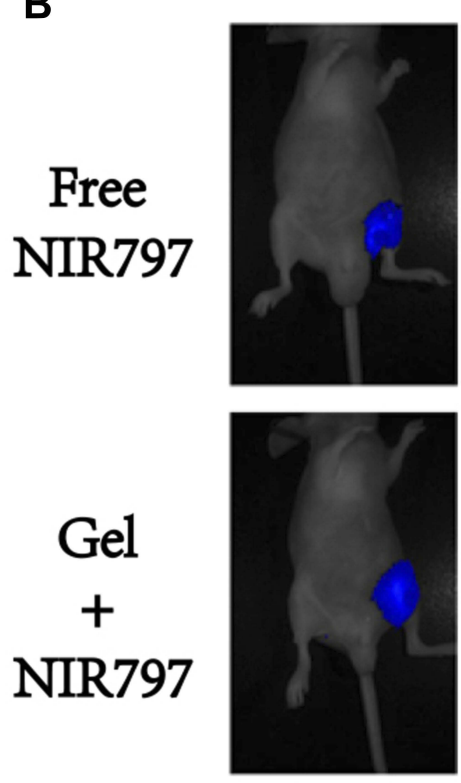

$1 \mathrm{~h}$
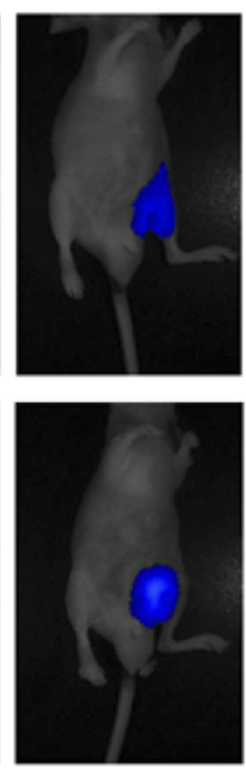

$6 \mathrm{~h}$
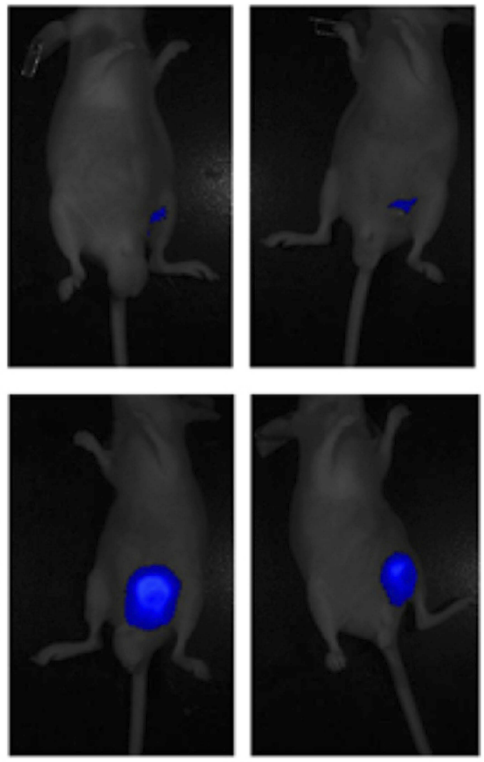

$24 \mathrm{~h}$

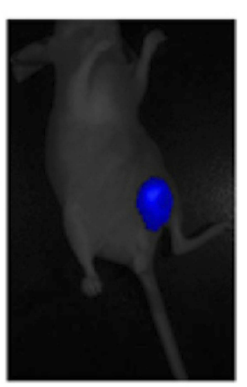

$48 \mathrm{~h}$
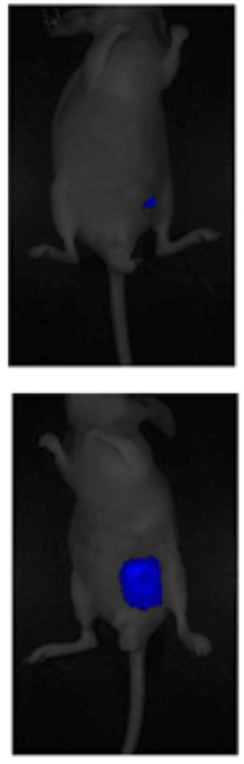

$72 \mathrm{~h}$
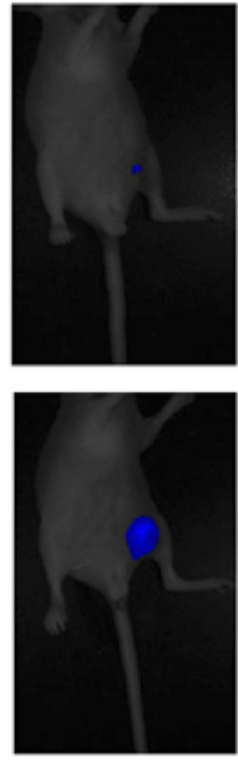

$96 \mathrm{~h}$

Figure 2 Degradability and controlled-release characteristics of HPC/SF/Gly hydrogels. (A) Photographs of HPC/SF/Gly hydrogels (200 $\mu \mathrm{L} / \mathrm{mice}$, 20\% glycerol concentration) in mice subcutaneously at different time points (Day 9, 21, 42, 60, 120). (B) In vivo near-infrared imaging of $0.1 \mathrm{~mL} \mathrm{NIR797} \mathrm{solution} \mathrm{(0.02} \mathrm{mg} / \mathrm{mL}$ ) and HPC/SF/Gly hydrogels ( $20 \%$ glycerol concentration) loaded with equivalent NIR797 in mice subcutaneously at different time points (I h, 6 h, 24 h, 48 h, 72 h, 96 h).

Abbreviations: HPC, hydroxypropyl cellulose; SF, silk fibroin; Gly, glycerol.

signal of NIR797 group weakened significantly after 24 $\mathrm{h}$ and was almost undetectable, Gel+NIR797 group's fluorescence did not change significantly at the initial $24 \mathrm{~h}$ and was significantly stronger than that of NIR797 group at and after $24 \mathrm{~h}$, indicating that $\mathrm{HPC} / \mathrm{SF} / \mathrm{Gly}$ hydrogels had good controlled-release characteristics.

\section{In vitro Effects of $i R G D$ on the Uptake of NPs by Tumor Cells}

After Rho-NPs and Rho-NPs+iRGD were co-incubated with tumor cells for $1 \mathrm{~h}$, respectively, the fluorescence intensity of tumor cells in both groups was measured by confocal imaging and flow cytometry. As shown in Figure 3A, the fluorescence intensity of tumor cells in Rho-NPs+iRGD group was higher, and the same result was also obtained by flow cytometry (Figure 3B), indicating that iRGD promoted the uptake of NPs by tumor cells.

\section{In vivo Distribution of HPC/SF/Gly Hydrogels Loaded with Different Drugs}

Figure 3C showed the changes in fluorescence intensity with time in mice after peritumoral injection of different NIR797 systems. While the fluorescence signals of all groups attenuated with time, NIR797 solution group showed the fastest attenuation. After $24 \mathrm{~h}$, the fluorescence intensity of NIR797 solution group was very weak, significantly lower than that of 
A

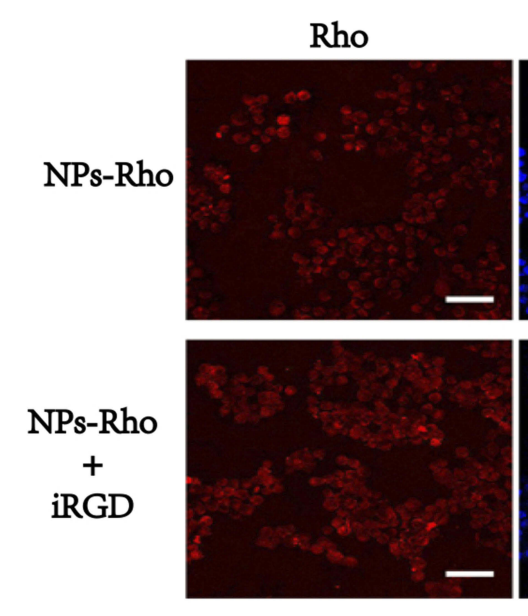

B

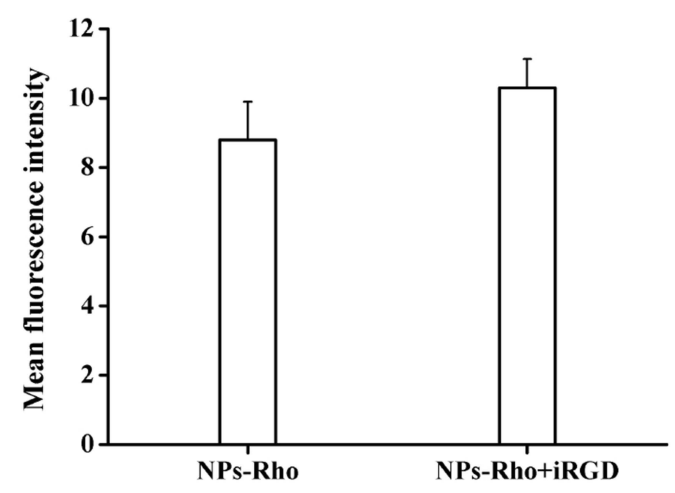

E

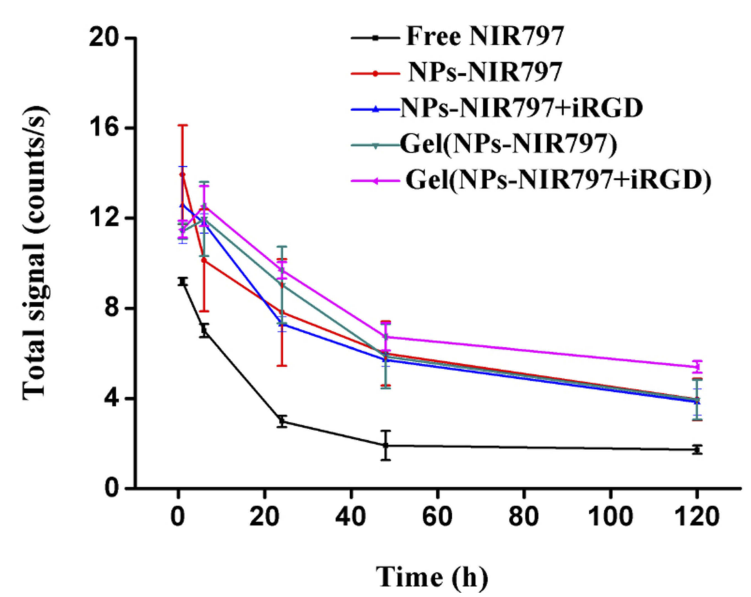

DAPI
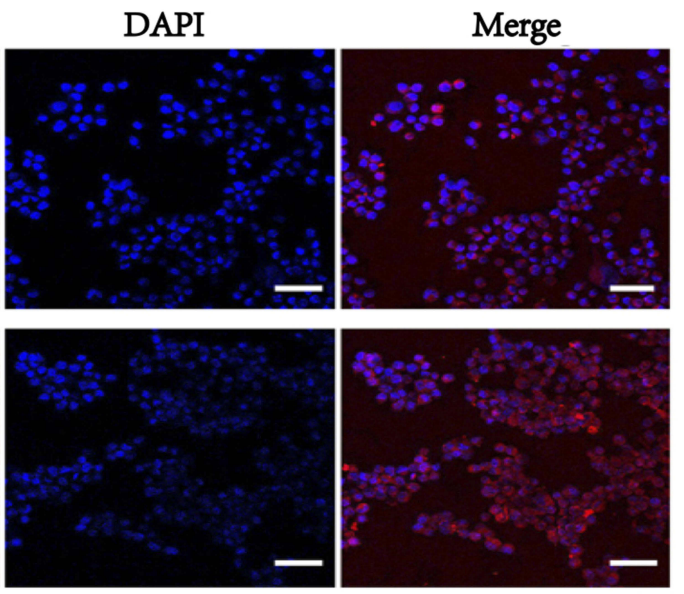

C

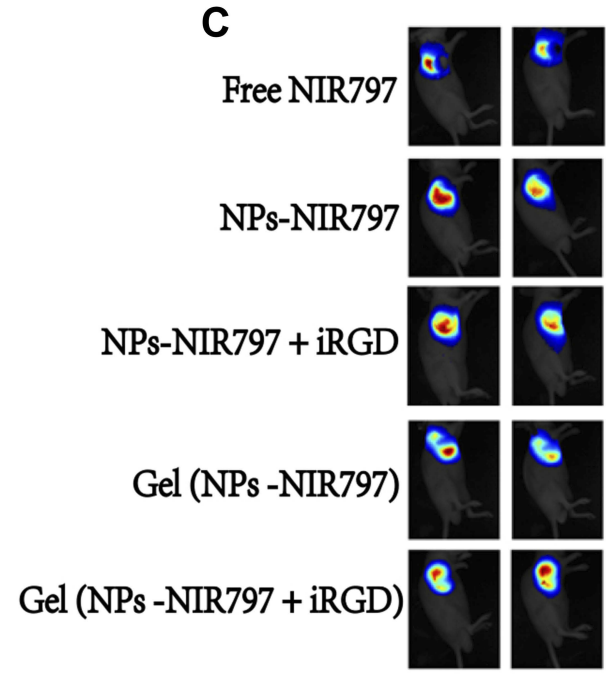

$1 \mathrm{~h}$
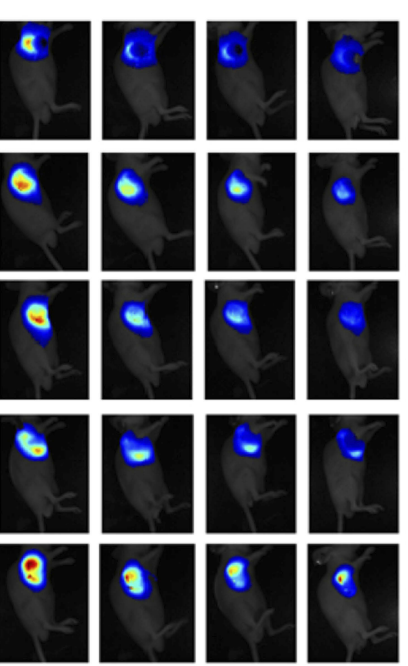

D
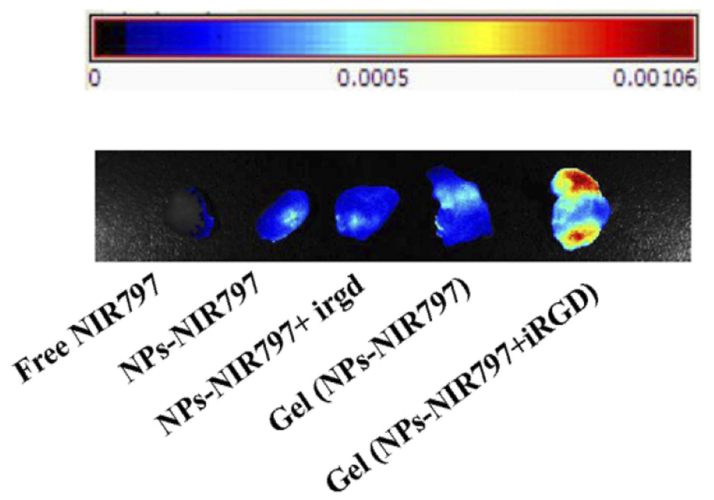

Figure 3 In vitro uptake assay and in vivo distribution of NPs. (A) Confocal photographs of Rho-NPs uptake by tumor cells (Scale Bar=50 $\mu$ m); (B) Flow cytometry quantification of Rho-NPs uptake by tumor cells. (C) and (E) Near-Infrared Imaging and quantitative data of distribution of NIR797-loaded different systems in mice; (D) Near-Infrared Imaging of distribution of NIR797-loaded different systems in tumor tissues.

Abbreviations: NPs, nanoparticles; Rho-NPs, nanoparticles loaded with Rhodamine B.

the other four groups, indicating that the NIR797 solution alone was quickly cleared in vivo. In other words, nanoparticles and hydrogels had strong retention ability. NIR797-NPs,
NIR797-NPs+iRGD and Gel (NIR797-NPs) groups showed similar fluorescence intensity, while Gel (NIR797-NPs + iRGD) showed relatively higher fluorescence intensity than 
the other groups. Figure 3E showed the quantitative data of fluorescence intensity of each group. The ratio of fluorescence intensity of Gel (NIR797-NPs+iRGD) to NIR797-NPs, NIR797-NPs+iRGD or Gel (NIR797-NPs) is approximately equal, about 1.4.

After $120 \mathrm{~h}$, the tumor tissues of each group were taken out for imaging. As shown in Figure 3D, there were almost no detectable fluorescence signals in the NIR797 group. The fluorescence intensity of NPs-NIR797, NPs-NIR797 + iRGD and Gel (NPs-NIR797) groups was similar. The fluorescence intensity of Gel (NPs-NIR797+iRGD) was

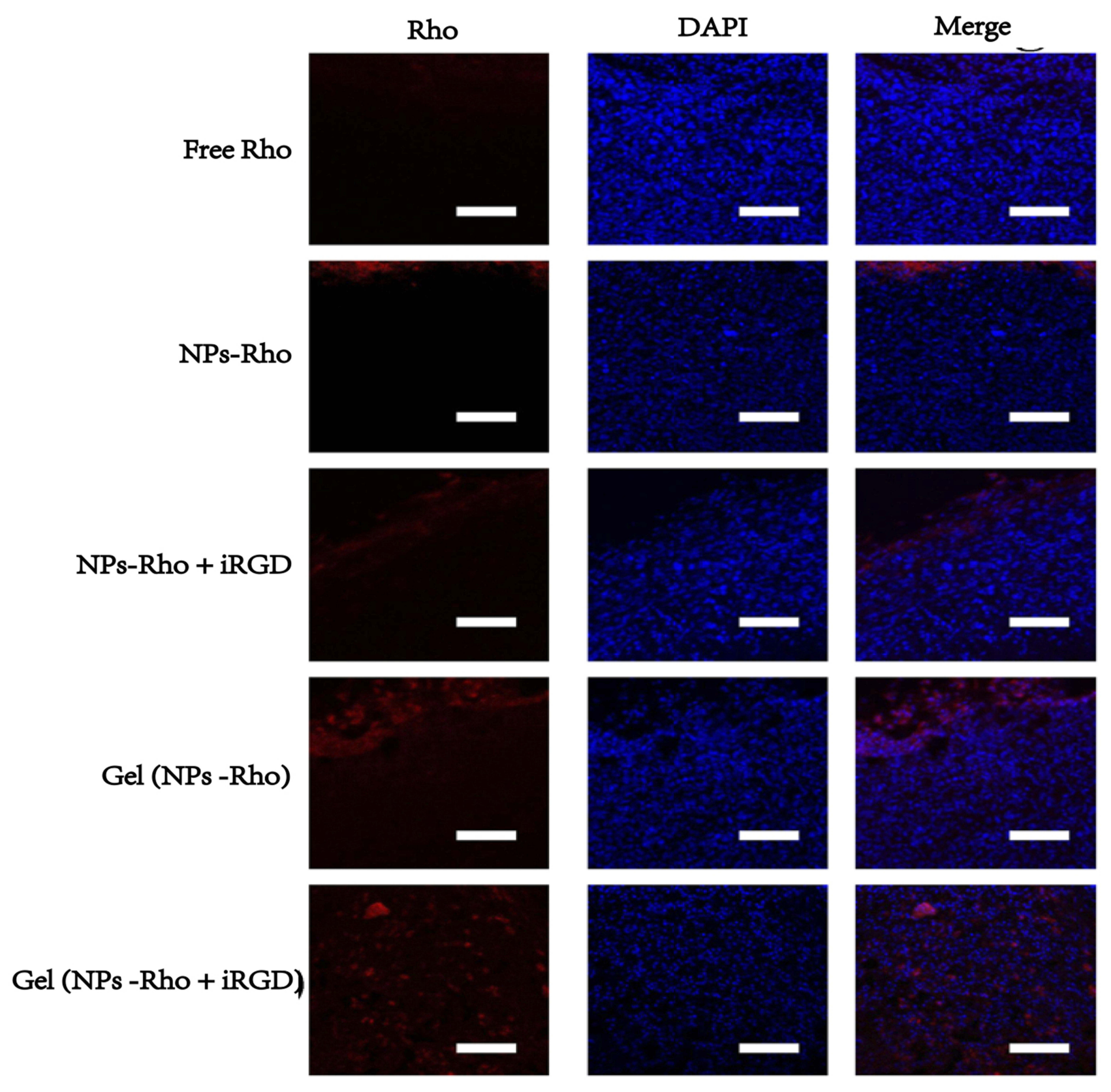

significantly higher than that of other groups. The ratio of Gel (NPs-NIR797+iRGD) to NPs-NIR797, NPs-NIR797 + iRGD or Gel (NPs-NIR797) was about 2.4, higher than in vivo (1.4).

Rho solution, Rho-NPs, Rho-NPs+iRGD, Gel (Rho-NPs) and Gel (Rho-NPs+iRGD) were injected into the peritumoral area of nude mice, respectively. Tumor tissues were taken out $120 \mathrm{~h}$ later and made into tissue sections. The distribution of microscopy. As shown in Figure 4, there was almost no fluorescence signal in the Rho solution group. Rho-NPs, Rho-loaded different systems was observed by confocal

Figure 4 Confocal imaging of Rho-loaded different systems in tumor tissues. Scale Bar $=100 \mu \mathrm{m}$. 
Rho-NPs+iRGD and Gel (Rho-NPs) were located at the edge of the tumor tissue, while Rho in Gel (Rho-NPs+iRGD) group was located inside the tumor tissue, indicating that NPs in Gel (Rho-NPs+iRGD) group had infiltrated into the tumor.

\section{In vivo Evaluation of Anti-Tumor Efficacy}

Compared with control group, the tumor volume of Free GA group, GA-NPs group, GA-NPs+iRGD group, Gel (GA-NPs) group, Gel (GA-NPs+iRGD) group, was 95\%, $70 \%, 70 \%, 59 \%, 31 \%$, respectively (Figure 5A) and the tumor weight was $96 \%, 70 \%, 67 \%, 62 \%, 34 \%$, respectively (Figure 5B). GA naked drug solution had no antitumor effect. GA-NPs, GA-NPs+iRGD and Gel (GA-NPs) showed moderate anti-tumor effects, significantly different from control group and Free GA group. In addition, Gel (GA-NPs) group showed the strongest anti-tumor effect.
Figure 5C was a photograph of nude mice tumors taken out on the 16th day. Tumors in Gel (GA-NPs) group were visibly smaller than other groups.

$\mathrm{HE}$ and Tunel staining were used to evaluate the level of necrosis and apoptosis of tumor cells, respectively. As shown in Figure 5D and E, compared with all other groups, Gel (GA-NPs+iRGD) showed the largest necrosis area and the highest level of apoptosis.

\section{Discussion}

Injectable hydrogels can be prepared by two methods: physical crosslinking and chemical crosslinking. During the chemical crosslinking process, the structure of loaded drugs or the activity of the loaded cells could be affected by some toxic crosslinking reagents or chemical heat generated by some reactions. Hence, the physical crosslinking method has been applied more widely. ${ }^{30,31}$ Temperature is
A
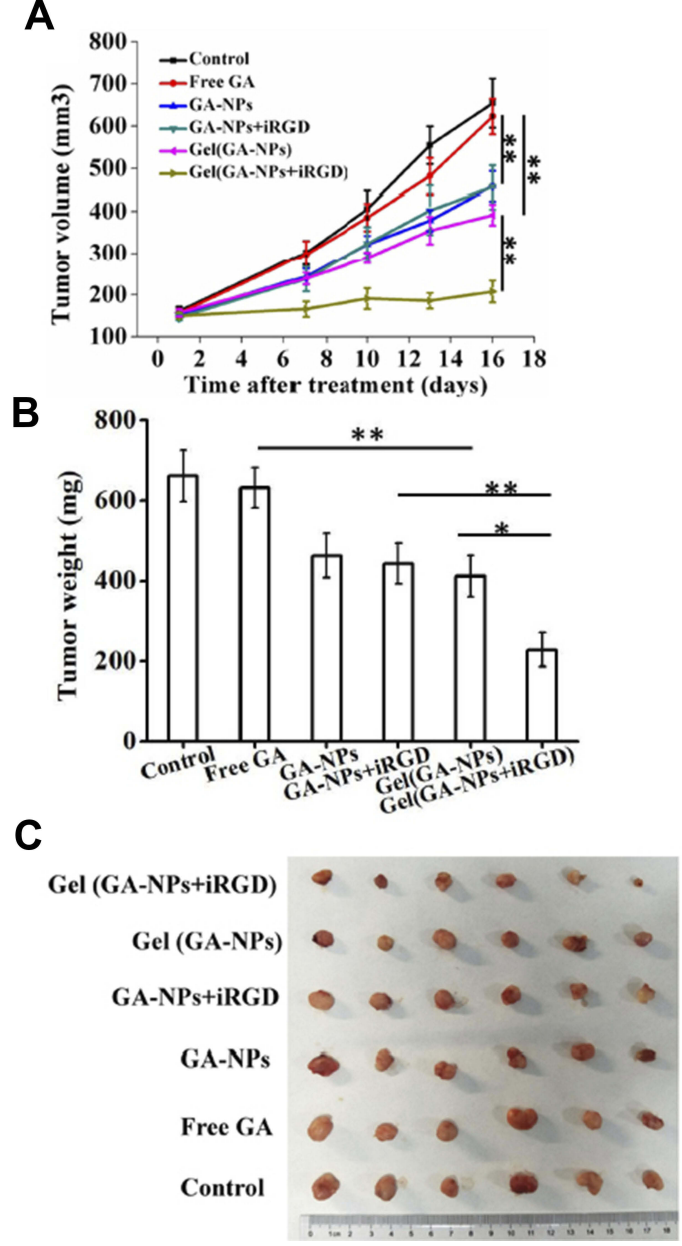

D

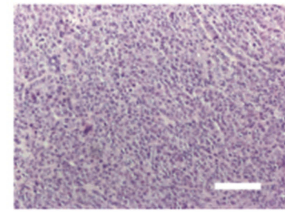

Control

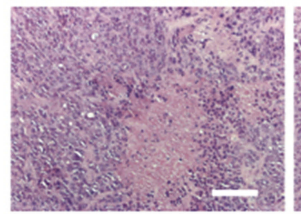

GA-NPs+iRGD

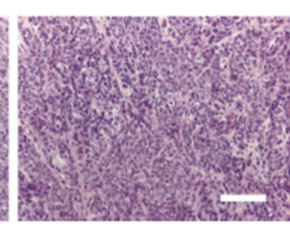

Free GA

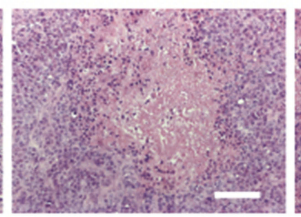

Gel(GA-NPs)

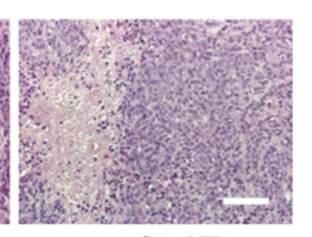

GA-NPs

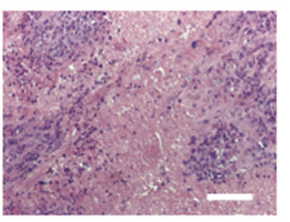

Gel(GA-NPs+iRGD)
E
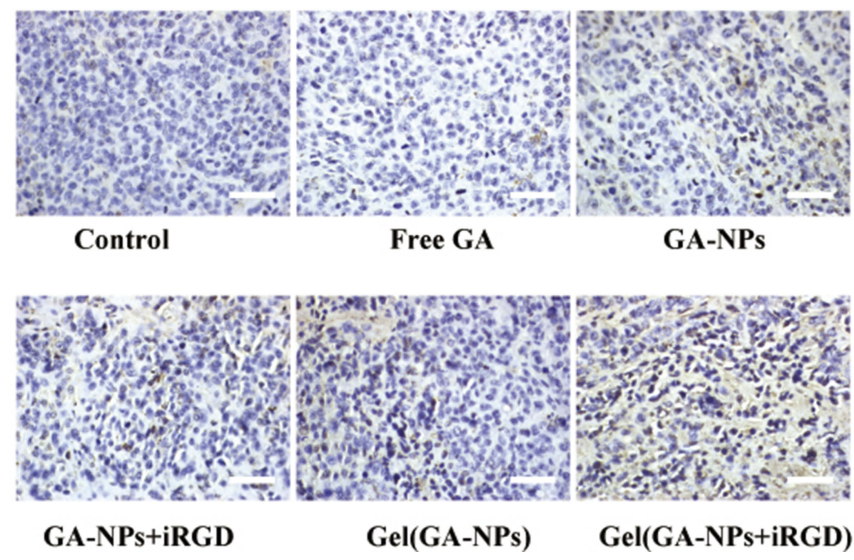

Figure 5 In vivo antitumor efficacy assay. (A) Changes in tumor volume in each group. (B) Tumor weight of mice in each group after 16 days. (C) Tumor photos on the 16th day; $* \mathrm{P}<0.05$, $* * \mathrm{P}<0.0 \mathrm{I}$. (D) HE (Scale Bar $=100 \mu \mathrm{m})$ and $(\mathbf{E})$ Tunel staining (Scale Bar $=50 \mu \mathrm{m})$ of tumor tissues in each group, evaluate the level of necrosis and apoptosis of tumor cells. 
one of the most common and simplest ways to prepare responsive injectable hydrogels. Temperature affects the physical state of the solution by influencing the hydrophobic interaction between hydrogen bonds and polymer molecules. $^{32}$ The lower critical solution temperature (LCST) of hydroxypropyl cellulose is $40^{\circ} \mathrm{C}$. When the solution of hydroxypropyl cellulose and silk fibroin is heated, the hydrophobicity between cellulose molecules is improved gradually with the increase of solution temperature, which induces the conversion of silk fibroin from irregular curling to beta-folding. Some small molecules, such as sodium chloride, calcium chloride and glycerol, can reduce the formation of polymer hydrate layer, reduce the LCST of polymer, and enhance the hydrophobic interaction between molecules. ${ }^{33}$ In our research, glycerol was added into the mixture of HPC and SF to evaluate glycerol's effect on gelation time of HPC-SF hydrogel. When glycerol was added, obvious turbidity appeared in HPC solution at $37^{\circ} \mathrm{C}$, which indicated that glycerol reduced LCST of hydroxypropyl cellulose. With the increase of glycerol concentration, not only the gelation time of hydrogel was shortened correspondingly, but also the micro-pore diameter is reduced. Perhaps with the increase of glycerol concentration, the hydrophobic interaction between cellulose molecules was enhanced gradually, which made silk fibroin more quickly change from irregular curling to beta-folding, resulting in shorter gelation time and higher crosslinking density.

Silk fibroin is one kind of degradable materials. Silk fibroin can be hydrolyzed by protease and the degradation products are mainly amino acids, which do not have toxicity. The biodegradation of silk fibroin is related to its molecular weight, structure, preparation conditions, pore size, protein concentration and host immunity. ${ }^{34}$ Hydroxypropyl cellulose is a natural cellulose approved by FDA and has good biodegradability and compatibility. ${ }^{35}$ Glycerol is commonly used as an additive in medicine and has been proven to be biocompatible in vivo. ${ }^{36}$ For example, Cardoso et al added glycerol as a component of the hydrogel in the preparation of calcium alginate gel, which proved that it had good biocompatibility in vivo. ${ }^{37}$ Our in vivo degradation experiments showed that the HPC/SF/Gly hydrogel was biodegradable and the degradation time was about 4 months. In addition, there was only a transient slight inflammatory reaction during the degradation process, which indicated that the hydrogel had good biocompatibility.

In our research, we chose a $20 \%$ glycerol hydrogel system, mainly because of its faster gel formation speed and smaller pore size. There are two main factors affecting the controlled release of hydrogels: pore size and biodegradation. The release of smaller-pore hydrogels is relatively slow, and the release of faster-degrading hydrogels is faster. ${ }^{38}$ In our research, we observed that the in vivo sustained release time of hydrogel was $96 \mathrm{~h}$, and the hydrogel was almost non-degradable during this period. Therefore, the pore size was the main factor affecting the sustained release performance of the hydrogel. Contrary to the rapid reduction of fluorescence signal in NIR797 solution group, the fluorescence signal of $\mathrm{HPC} / \mathrm{SF} / \mathrm{Gly}$ hydrogel with a pore size of $400 \mathrm{~nm}$ showed a slow decrease, indicating that the hydrogel had a good controlled release performance. In addition, it is intuitive, simple and convenient to use experimental gel directly loaded with fluorescence small molecules.

Gambogic acid is an effective drug for cancer treatment, but its poor water solubility and serious side effects hampered the clinical application. In recent years, some nanopolymers have partly improved the therapeutic effect of gambogic acid by improving its water solubility and changing its biological distribution. ${ }^{16,17}$ Among various nanomaterials, PEG-PCL diblock copolymers have been widely used in cancer drug delivery due to their good biocompatibility, biodegradability and safety. ${ }^{39,40}$ Recently, it has been reported that peritumoral injection of PEG-PCL nanoparticles loaded with gambogic acid improves the anti-tumor effect compared with naked drug group. ${ }^{41}$

iRGD has been widely used in the research of cancer treatment in recent years due to its ability to increase the infiltration of drugs and drug-loaded nanoparticles into tumors. $^{42,43}$ Its tumour osmotic effect involves many steps, including binding to integrin receptors, protease hydrolysis exposing C-terminal motifs, binding to NRP1 , and eventually leading to increased permeability of cells and tissues. Therefore, iRGD-modified compounds can accumulate in tumour tissues, thereby improving the therapeutic effect. Recent studies have found that not only iRGD-modified compounds, but also physically mixed iRGD-compounds can increase tumor infiltration and enhance anti-tumor effects. ${ }^{20,44}$ However, physical mixing often requires multiple doses, ${ }^{24,25}$ possibly because iRGD is a short hydrophilic peptide with a short half-life.

As a controlled release carrier, hydrogels have been widely used to deliver drugs, proteins, polypeptides, etc. $^{45,46}$ We have prepared a kind of injectable hydrogels with excellent controlled-release performance, good biocompatibility and biodegradability. Physically mix it with 
iRGD. On the one hand, it can prolong the retention time of iRGD and avoid repeated injection through the sustained-release effect of the hydrogels; on the other hand, it can avoid the complexity of chemical bonding and the possible harm to protein function during the bonding process.

In this study, we prepared HPC/SF/Gly drug delivery system loaded with both water-soluble PEG-PCL nanoparticles of gambogic acid and iRGD. In vitro drug-releasing experiments showed $20 \%$ of GA release and $60 \%$ of iRGD release from $\mathrm{HPC} / \mathrm{SF} / \mathrm{Gly}$ hydrogel. It is an advantage that the drug in our designed hydrogel released much more slowly. They could play their roles for a longer time in acid tumor microenvironment. In vitro tumor cell uptake experiments showed that iRGD significantly promoted the uptake of nanoparticles by tumor cells. In vivo nanoparticle penetration experiments showed that the nanoparticles loaded by Gel (NPs+iRGD) penetrated into the core site of tumor tissue, while the nanoparticles in NPs, NPs + iRGD and Gel (NPs) group only located at the edge of the tumor tissue. The possible reason is the osmotic effect of iRGD. Although there is iRGD in NPs+iRGD group, it did not show good penetration ability because of its rapid clearance by the body. Hydrogels prolong the retention time of iRGD around tumors through its sustained release, thus promoting the infiltration of NPs into tumors. The infiltration ability of nanoparticles in different groups also explained the difference of near infrared fluorescence intensity. In addition, the difference of near-infrared imaging results between Gel (NPs-NIR797 + iRGD) group and other nanoparticles group, of tumors is more obvious than that of the whole body. Perhaps in vivo near-infrared imaging, besides the fluorescence intensity of tumors, the fluorescence intensity of peritumoral tissue also accounts for a part of the proportion.

The difference of anti-tumor activity among groups in vivo can be well explained by the results of near-infrared and confocal imaging. Compared with the control group, the Free GA group showed mild anti-tumor activity, which was due to the rapid elimination of gambogic in vivo. Although most of the nanoparticles in Gel (GA-NPs), GA-NPs and NPs $+\mathrm{iRGD}$ group were distributed in the peritumoral tissues, they showed a significantly inhibitory effect of tumor growth. Gel (GA-NPs+iRGD) group had the strongest anti-tumor effect because of more gambogic acid infiltrating into the tumors. In addition, although the imaging results showed that the fluorescence intensity and location distribution of nanoparticles of GA-NPs+iRGD, GA-NPs and NPs group were similar, the anti-tumor ability of Gel (GA-NPs) group was slightly stronger than that of both GA-NPs and NPs+iRGD group in vivo. It may be due to the sustained-release performance of $\mathrm{HPC} / \mathrm{SF} /$ Gly hydrogel. The results of HE and TUNEL in tumor tissues were consistent with the trend of anti-tumor experiment, which further confirmed the anti-tumor activity of each group in pathology. Finally, we conclude that Gel (GA-NPs + iRGD) exerts an effective anti-tumor effect in three aspects: 1. retention effect, unlike naked drugs which could be quickly cleared in vivo; 2 . local administration, to a certain extent, increasing the drug concentration of tumors; 3 . continuous sustained release of iRGD promoting the penetration of NPs into the deep part of tumors and further increasing the drug concentration in tumors.

Local chemotherapy can not only increase the anti-tumor effect of drugs, but also reduce systemic toxicity. ${ }^{4,48}$ In our safety assessment experiments, there were no obvious side effects of GA and the formulation. The injectable hydrogels packaging GA-NPs and iRGD allowed a relatively small dose of GA due to great penetration ability, which accounting for the reduction of systemic toxicity.

\section{Conclusions}

In our research, we constructed a new thermosensitive injectable hydrogel by the physical mixing of HPC, SF and glycerol. With the increase of temperature, HPC can change the structure of silk fibroin to gel through its enhanced hydrophobic interaction between molecules. At the same time, glycerol can further enhance the hydrophobicity of cellulose and accelerate the gelling speed and crosslinking density. The prepared HPC/SF/Gly hydrogels have the advantages of short gelling time, good compatibility and sustained release.

Then, we used HPC/SF/Gly hydrogels to load tumor osmotic peptide iRGD and gambogic acid nanoparticles. Through its controlled-release effect, we prolonged the retention time of $\mathrm{iRGD}$ and promoted the penetration of nanoparticles into the deep part of tumors, thus playing an effective anti-tumor effect without obvious side effects. In addition, the method is simple and operable, and physical mixing avoids the complexity of chemical bonding and the possible harm to protein functions during the chemical bonding process. This kind of hydrogels can serve as a promising drug delivery system.

\section{Acknowledgments}

Qin Liu is supported by the National Natural Science Foundation of China (No. 81972309, No. 81302053), 
Jiangsu Provincial Medical Youth Talent (No. QNRC 2016045) and Supported by the Fundamental Research Funds for the Central Universities (No. 021414380450). Quanan Zhang is supported by a grant from the Natural Science Foundation of Jiangsu Province (No. BK 20161110). The funding sources had no role in the study design, data collection, data analysis, data interpretation, or writing of the report.

\section{Disclosure}

The authors report no conflicts of interest in this work.

\section{References}

1. Song $\mathrm{Z}$, Wu Y, Yang J, et al. Progress in the treatment of advanced gastric cancer. Tumour Biol. 2017;39(7):1010428317714626.

2. Gatti-Mays ME, Redman JM, Collins JM, et al. Cancer vaccines: enhanced immunogenic modulation through therapeutic combinations. Hum Vaccin Immunother. 2017;13(11):2561-2574. doi:10.1080/2164 5515.2017.1364322

3. Liu J, Pang Y, Zhang S, et al. Triggerable tough hydrogels for gastric resident dosage forms. Nat Commun. 2017;8(1):124. doi:10.1038/s41467017-00144-z

4. Dimatteo R, Darling NJ, Segura T. In situ forming injectable hydrogels for drug delivery and wound repair. Adv Drug Deliv Rev. 2018;127:167-184. doi:10.1016/j.addr.2018.03.007

5. Mirdailami O, Soleimani M, Dinarvand R, et al. Controlled release of rhEGF and rhbFGF from electrospun scaffolds for skin regeneration. J Biomed Mater Res A. 2015;103(10):3374-3385. doi:10.1002/jbm. a.35479

6. Norouzi M, Shabani I, Ahvaz HH, et al. PLGA/gelatin hybrid nanofibrous scaffolds encapsulating EGF for skin regeneration. $J$ Biomed Mater Res A. 2015;103(7):2225-2235. doi:10.1002/jbm.a.35355

7. Das V, Bruzzese F, Konecny P, et al. Pathophysiologically relevant in vitro tumor models for drug screening. Drug Discov Today. 2015;20(7):848-855. doi:10.1016/j.drudis.2015.04.004

8. Sun W, Incitti T, Migliaresi C, et al. Viability and neuronal differentiation of neural stem cells encapsulated in silk fibroin hydrogel functionalized with an IKVAV peptide. J Tissue Eng Regen Med. 2017;11(5):1532-1541. doi:10.1002/term.2053

9. Floren M, Bonani W, Dharmarajan A, et al. Human mesenchymal stem cells cultured on silk hydrogels with variable stiffness and growth factor differentiate into mature smooth muscle cell phenotype. Acta Biomater. 2016;31:156-166. doi:10.1016/j.actbio.2015.11.051

10. Lovett ML, Wang X, Yucel T, et al. Silk hydrogels for sustained ocular delivery of anti-vascular endothelial growth factor (anti-VEGF) therapeutics. Eur J Pharm Biopharm. 2015;95(Pt B):271-278. doi:10.1016/j.ejpb.2014.12.029

11. Li C, Qi Q, Lu N, et al. Gambogic acid promotes apoptosis and resistance to metastatic potential in MDA-MB-231 human breast carcinoma cells. Biochem Cell Biol. 2012;90(6):718-730. doi:10. 1139/02012-030

12. Zou ZY, Wei J, Li XL, et al. Enhancement of anticancer efficacy of chemotherapeutics by gambogic acid against gastric cancer cells. Cancer Biother Radiopharm. 2012;27(5):299-306. doi:10.1089/cbr.2010.0943

13. Park MS, Kim NH, Kang CW, et al. Antimetastatic effects of gambogic acid are mediated via the actin cytoskeleton and NF-kappaB pathways in SK-HEP1 cells. Drug Dev Res. 2015;76(3):132-142. doi:10.1002/ddr.21249

14. Huang J, Zhu X, Wang H, et al. Role of gambogic acid and NaI(131) in A549/DDP cells. Oncol Lett. 2017;13(1):37-44. doi:10.3892/ ol.2016.5435
15. Zhang Y, Yang Z, Tan X, et al. Development of a more efficient albumin-based delivery system for gambogic acid with low toxicity for lung cancer therapy. AAPS PharmSciTech. 2017;18(6):1987-1997. doi:10.1208/s12249-016-0670-4

16. Yao J, Li Y, Sun X, et al. Nanoparticle delivery and combination therapy of gambogic acid and all-trans retinoic acid. Int J Nanomedicine. 2014;9:3313-3324. doi:10.2147/IJN.S62793

17. Doddapaneni R, Patel K, Owaid IH, et al. Tumor neovasculature-targeted cationic PEGylated liposomes of gambogic acid for the treatment of triple-negative breast cancer. Drug Deliv. 2016;23(4):1232-1241. doi:10.3109/10717544.2015.1124472

18. Yan X, Yang Y, He L, et al. Gambogic acid grafted low molecular weight heparin micelles for targeted treatment in a hepatocellular carcinoma model with an enhanced anti-angiogenesis effect. Int J Pharm. 2017;522 (1-2):110-118. doi:10.1016/j.ijpharm.2017.02.051

19. Thambi T, Park JH. Recent advances in shell-sheddable nanoparticles for cancer therapy. J Biomed Nanotechnol. 2014;10(9):1841-1862. doi:10.1166/jbn.2014.1977

20. Sugahara KN, Teesalu T, Karmali PP, et al. Coadministration of a tumor-penetrating peptide enhances the efficacy of cancer drugs. Science (New York, NY). 2010;328(5981):1031-1035. doi:10.1126/ science. 1183057

21. Domingo-Espin J, Petegnief V, de Vera N, et al. RGD-based cell ligands for cell-targeted drug delivery act as potent trophic factors. Nanomedicine. 2012;8(8):1263-1266. doi:10.1016/j.nano.2012.06.005

22. Lagunas A, Comelles J, Martinez E, et al. Cell adhesion and focal contact formation on linear RGD molecular gradients: study of non-linear concentration dependence effects. Nanomedicine. 2012;8 (4):432-439. doi:10.1016/j.nano.2011.08.001

23. Ruoslahti E. Peptides as targeting elements and tissue penetration devices for nanoparticles. Adv Mater. 2012;24(28):3747-3756. doi:10.1002/adma.v24.28

24. Sugahara KN, Scodeller P, Braun GB, et al. A tumor-penetrating peptide enhances circulation-independent targeting of peritoneal carcinomatosis. J Control Release. 2015;212:59-69. doi:10.1016/j. jconrel.2015.06.009

25. Puig-Saus C, Rojas LA, Laborda E, et al. iRGD tumor-penetrating peptide-modified oncolytic adenovirus shows enhanced tumor transduction, intratumoral dissemination and antitumor efficacy. Gene Ther. 2014;21(8):767-774. doi:10.1038/gt.2014.52

26. Wang K, Zhang X, Liu Y, et al. Tumor penetrability and anti-angiogenesis using iRGD-mediated delivery of doxorubicin-polymer conjugates. Biomaterials. 2014;35(30):8735-8747. doi:10.1016/j.biomaterials.2014. 06.042

27. Akashi Y, Oda T, Ohara Y, et al. Anticancer effects of gemcitabine are enhanced by co-administered iRGD peptide in murine pancreatic cancer models that overexpressed neuropilin-1. $\mathrm{Br} J$ Cancer. 2014;110(6):1481-1487. doi:10.1038/bjc.2014.49

28. Schmithals C, Koberle V, Korkusuz H, et al. Improving drug penetrability with iRGD leverages the therapeutic response to sorafenib and doxorubicin in hepatocellular carcinoma. Cancer Res. 2015;75 (15):3147-3154. doi:10.1158/0008-5472.CAN-15-0395

29. Cun X, Chen J, Ruan S, et al. A novel strategy through combining iRGD peptide with tumor-microenvironment-responsive and multistage nanoparticles for deep tumor penetration. ACS Appl Mater Interfaces. 2015;7(49):27458-27466. doi:10.1021/acsami.5b09391

30. Kanikkannan N. Iontophoresis-based transdermal delivery systems. BioDrugs. 2002;16(5):339-347. doi:10.2165/00063030-20021605000003

31. Karavasili C, Fatouros DG. Smart materials: in situ gel-forming systems for nasal delivery. Drug Discov Today. 2016;21(1):157-166. doi:10.1016/j.drudis.2015.10.016

32. Elias PZ, Liu GW, Wei H, et al. A functionalized, injectable hydrogel for localized drug delivery with tunable thermosensitivity: synthesis and characterization of physical and toxicological properties. J Control Release. 2015;208:76-84. doi:10.1016/j.jconrel.2015.03.003 
33. Almeida N, Rakesh L, Zhao J. Phase behavior of concentrated hydroxypropyl methylcellulose solution in the presence of mono and divalent salt. Carbohydr Polym. 2014;99:630-637. doi:10.1016/ j.carbpol.2013.08.081

34. Li M, Ogiso M, Minoura N. Enzymatic degradation behavior of porous silk fibroin sheets. Biomaterials. 2003;24(2):357-365. doi:10.1016/S0142-9612(02)00326-5

35. Hoo SP, Sarvi F, Li WH, et al. Thermoresponsive cellulosic hydrogels with cell-releasing behavior. ACS Appl Mater Interfaces. 2013;5 (12):5592-5600. doi:10.1021/am4009133

36. Sugawara A, Fujikawa K, Hirayama S, et al. In vivo characteristics of premixed calcium phosphate cements when implanted in subcutaneous tissues and periodontal bone defects. J Res Natl Inst Stand Technol. 2010;115(4):277-290. doi:10.6028/jres.115.021

37. Cardoso DA, van den Beucken JJ, Both LL, et al. Gelation and biocompatibility of injectable alginate-calcium phosphate gels for bone regeneration. J Biomed Mater Res A. 2014;102(3):808-817. doi:10.1002/jbm.a.34754

38. Zhong T, Jiang Z, Wang P, et al. Silk fibroin/copolymer composite hydrogels for the controlled and sustained release of hydrophobic/ hydrophilic drugs. Int J Pharm. 2015;494(1):264-270. doi:10.1016/j. ijpharm.2015.08.035

39. Shen Y, Leng M, Yu H, et al. Effect of amphiphilic PCL-PEG nano-micelles on HepG2 cell migration. Macromol Biosci. 2015;15 (3):372-384. doi:10.1002/mabi.201400376

40. Xu L, Xu X, Chen H, et al. Ocular biocompatibility and tolerance study of biodegradable polymeric micelles in the rabbit eye. Colloids Surf B Biointerfaces. 2013;112:30-34. doi:10.1016/j.colsurfb.2013.06.047

41. Zhang D, Zou Z, Ren W, et al. Gambogic acid-loaded PEG-PCL nanoparticles act as an effective antitumor agent against gastric cancer. Pharm Dev Technol. 2018;23(1):33-40. doi:10.1080/ 10837450.2017 .1295068
42. Hamilton AM, Aidoudi-Ahmed S, Sharma S, et al. Nanoparticles coated with the tumor-penetrating peptide iRGD reduce experimental breast cancer metastasis in the brain. J Mol Med (Berl). 2015;93 (9):991-1001. doi:10.1007/s00109-015-1279-X

43. Deng C, Zhang Q, Fu Y, et al. Coadministration of oligomeric hyaluronic acid-modified liposomes with tumor-penetrating peptide-iRGD enhances the antitumor efficacy of doxorubicin against melanoma. ACS Appl Mater Interfaces. 2017;9(2):1280-1292. doi:10.1021/acsami.6b13738

44. Sugahara KN, Teesalu T, Karmali PP, et al. Tissue-penetrating delivery of compounds and nanoparticles into tumors. Cancer Cell. 2009;16(6):510-520. doi:10.1016/j.ccr.2009.10.013

45. St'astny M, Plocova D, Etrych T, et al. HPMA-hydrogels result in prolonged delivery of anticancer drugs and are a promising tool for the treatment of sensitive and multidrug resistant leukaemia. Eur J Cancer. 2002;38(4):602-608. doi:10.1016/S0959-8049(01)00421-X

46. He H, Grignol V, Karpa V, et al. Use of a nanoporous biodegradable miniature device to regulate cytokine release for cancer treatment. J Control Release. 2011;151(3):239-245. doi:10.1016/j.jconrel.2011. 02.020

47. Bastiancich C, Danhier P, Preat V, et al. Anticancer drug-loaded hydrogels as drug delivery systems for the local treatment of glioblastoma. J Control Release. 2016;243:29-42. doi:10.1016/j. jconrel.2016.09.034

48. Krukiewicz K, Zak JK. Biomaterial-based regional chemotherapy: local anticancer drug delivery to enhance chemotherapy and minimize its side-effects. Mater Sci Eng C Mater Biol Appl. 2016;62:927-942. doi:10.1016/j.msec.2016.01.063
International Journal of Nanomedicine

\section{Publish your work in this journal}

The International Journal of Nanomedicine is an international, peerreviewed journal focusing on the application of nanotechnology in diagnostics, therapeutics, and drug delivery systems throughout the biomedical field. This journal is indexed on PubMed Central, MedLine, CAS, SciSearch ${ }^{\circledR}$, Current Contents ${ }^{\mathbb{R}} /$ Clinical Medicine,
Journal Citation Reports/Science Edition, EMBase, Scopus and the Elsevier Bibliographic databases. The manuscript management system is completely online and includes a very quick and fair peer-review system, which is all easy to use. Visit http://www.dovepress.com/ testimonials.php to read real quotes from published authors. 